\title{
Design and Simulation of Indoor Position System Based on PDR and Least Square RSSI Estimation
}

\author{
Jiahui $\mathrm{Lu}^{1, \mathrm{a}}$, Zhigang Wen ${ }^{2, \mathrm{~b}}$ \\ ${ }^{1}$ School of Electronic Engineering, Beijing University of Posts and Telecommunications, Beijing, \\ China \\ ${ }^{2}$ Institute of Sensing Technology and Business, BUPT, Wuxi, China \\ aemail: lujiahui11@126.com, bzwen@bupt.edu.cn
}

Keywords: Indoor Position System, PDR, Least Square RSSI Estimation, and RSSI Position Algorithm

\begin{abstract}
In this paper, we at first proposed a Least Square RSSI Estimation algorithm for the indoor wireless position. Next, a wireless indoor position system is designed based on the proposed algorithm and the PDR (Pedestrian Dead Reckoning) is used to correct the deviation of RSSI indoor position algorithm in this system. The simulations showed that the proposed method has a 34\% accuracy improvement over systems based on the traditional RSSI indoor position algorithm. The proposed system provides a new algorithm for the indoor wireless position.
\end{abstract}

\section{Introduction}

At present, GPS shows the good performance of outdoors position. However, GPS can't cover the indoor environment because the building weakens the signal of GPS. There are several methods used for Indoor Positioning: Wi-Fi fingerprint, RFID, Infrared, Bluetooth, Ultra-Wideband. Infrared positioning only applies to short distance and it is easily affected by fluorescent or indoor lighting. RFID and Ultra-Wideband has a high demand of indoor position hardware. Wi-Fi fingerprint can be divided into two sections: offline fingerprint collection section and online positioning section, and Wi-Fi fingerprint has to maintain and update a Wi-Fi fingerprint database, moreover the calculation complexity is unbearable when the number of the AP grows.

Apple put forwards iBeacon at 2013 WWDC. Currently, the Apple devices, such as iPhone and iPad equipped with iOS 7.0 and Bluetooth 4.0 can use iBeacon. With the help of iBeacon and the development of mobile terminal, indoor position accuracy has been greatly improved. Thus, this paper puts forward a newly indoor position algorithm: Least Square RSSI Estimation.

Except the indoor position algorithm stated above, Inertial Positioning Method has been paid more and more attention to. And this paper uses PDR technology to correct and deviation of RSSI Indoor Position Algorithm and combines PDR and Least Square RSSI Estimation to improve the accuracy of the indoor position system.

\section{Disadvantage of Triangle Centroid Location Algorithm}

RSSI Indoor Positioning Algorithm is based on signal propagation model. In the electromagnetic wave propagation process, the signal strength attenuates when the propagation distance grows. Under ideal conditions, signal propagation model can be expressed as:

$$
p=p_{0}+10 n \lg \left(\frac{d}{d_{0}}\right)
$$

$\mathrm{d}_{0}$ represents specified distance, $\mathrm{p} 0$ represents the signal strength at specified distance, $\mathrm{d}$ represents the real distance, $\mathrm{n}$ represents propagation parameter, which can be get in the measurement.

Let $\mathrm{d} 0=1$, type $(1)$ can be simplified as:

$$
p=p_{0}+10 n \lg (d)
$$


We can get the distances between mobile terminal and iBeacon through signal propagation model and this is the foundation of Triangle Centroid Location Algorithm. Triangle Centroid Location Algorithm can be expressed as Figure.1. In Figure.1, AP1, AP2, AP3 represents iBeacon, $\mathrm{d} 11, \mathrm{~d} 12$, d13 represents the distance between mobile terminal and iBeacon. According to Triangle Centroid Location Algorithm, three iBeacons can get coordinate of the mobile terminal.

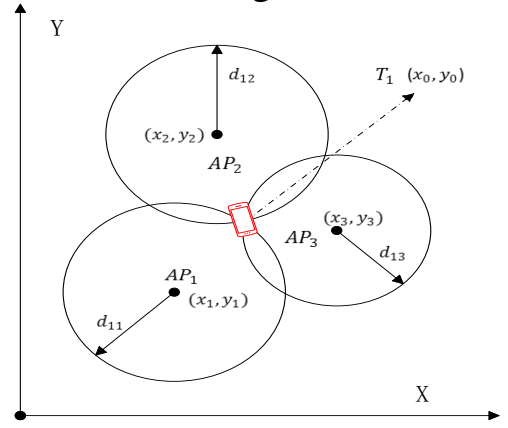

Fig.1. Triangle Centroid Location Algorithm

But Figure 1 is the most ideal condition, but in fact, unfortunately, the triangle centroid location algorithm can't get the mobile terminal location when intersection points of this three circle can't make a triangle or this three circles don't intersect with each other, thus Triangle Centroid Location Algorithm can't get the coordinate of the mobile terminal. This condition can be showed as Figure 2:
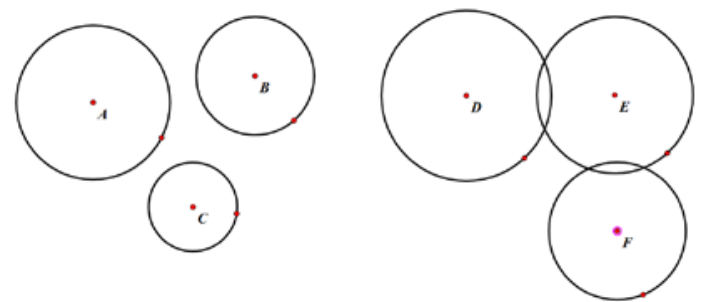

Fig.2. The instance that Triangle Centroid Location Algorithm doesn't work

Moreover, Triangle Centroid Location Algorithm doesn't regard the whole iBeacon network as an entirety. The contact of the iBeacon network can't be reflected in the triangle centroid location algorithm because the algorithm separates the every three iBeacons into groups.

\section{The Least Square RSSI Estimation}

The least square method can be applied to indoor position system. Provided that the mobile terminal has received the signal from $\mathrm{n}$ iBeacons, by these signals and the signal propagation model we can get the distances between mobile terminal and the iBeacons, we represent these distance as $\mathrm{d} 1, \mathrm{~d} 2, \ldots \mathrm{dn}$ and the coordinates of iBeacons as $(\mathrm{x} 1, \mathrm{y} 1),(\mathrm{x} 2, \mathrm{y} 2) \ldots(\mathrm{xn}, \mathrm{yn})$. If the coordinate of mobile terminal is $(\mathrm{x}, \mathrm{y})$, we can easily get the type(3).

$$
\left\{\begin{array}{c}
\left(x-x_{1}\right)^{2}+\left(y-y_{1}\right)^{2}=d_{1}^{2} \\
\left(x-x_{2}\right)^{2}+\left(y-y_{2}\right)^{2}=d_{2}^{2} \\
\cdots \cdots \\
\left(x-x_{n}\right)^{2}+\left(y-y_{n}\right)^{2}=d_{n}^{2}
\end{array}\right.
$$

Let:

$$
R^{2}=x^{2}+y^{2} \text { And }\left\{\begin{array}{c}
r_{1}^{2}=d_{1}^{2}-x_{1}^{2}-y_{1}^{2} \\
r_{2}^{2}=d_{2}^{2}-x_{2}^{2}-y_{2}^{2} \\
\cdots \cdots \\
r_{n}^{2}=d_{n}^{2}-x_{n}^{2}-y_{n}^{2}
\end{array}\right.
$$

Type (3) can be represented as follow: 


$$
\left\{\begin{array}{c}
-2 x_{1} x-2 y_{1} y+R^{2}=r_{1}^{2} \\
-2 x_{2} x-2 y_{2} y+R^{2}=r_{2}^{2} \\
\cdots \cdots \\
-2 x_{n} x-2 y_{n} y+R^{2}=r_{n}^{2}
\end{array}\right.
$$

In type (4), $x$ and $y$ is unknown number. In indoor position system, the numbers of the signals that can be received by the mobile terminal are mostly more than two. In this situation, number of the equations is more than the number of unknown number, thus the equations is over-determined equations and the equations can't get the exact solutions. In this condition, the solution created by the least square method can minimizes the sum of squared residuals, and the solution is called Least Squares Solutions of over-determined equations.

Let:

$$
x=\left[\begin{array}{c}
x \\
y \\
R^{2}
\end{array}\right], A=\left[\begin{array}{ccc}
-2 x_{1} & -2 y_{1} & 1 \\
-2 x_{2} & -2 y_{2} & 1 \\
\ldots \ldots & \\
-2 x_{n} & -2 y_{n} & 1
\end{array}\right], b=\left[\begin{array}{c}
r_{1}^{2} \\
r_{2}^{2} \\
\cdots \ldots . . \\
r_{n}^{2}
\end{array}\right]
$$

Type (4) can be represented as follow:

$A x=b$

According to existence and uniqueness theorem of least square solution, if $\operatorname{rank}(A)=m<n$, the least square solution is unique and can be represented as follow:

$\hat{x}=\left(A^{T} A\right)^{-1} A^{T} b$

From type (7), we can get the solution of coordinate (x,y). And this solution can minimize the sum of squared residuals.

\section{The Weighted Least Square RSSI Estimation}

In the process of electromagnetic wave propagation, because of Multipath effect, Shadowing effect, obstacle, the signal of iBeacon may fluctuate, which may reduce the accuracy of indoor position system. In practice, we can easily find that the shorter the distance between the iBeacon and the mobile terminal is, the stronger signal intensity the mobile terminal can get, the more precisely the signal propagation model can work. The reason is obvious: when the distance is shorter, the iBeacon signal receives less interference, the distance measurement is more precise. So every iBeacon in the system are not equals, in this condition, we introduce Weighted Least Square RSSI Estimation. The solution of weighted least square RSSI Estimation is:

$$
\hat{x}=\left(A^{T} W A\right)^{-1} A^{T} W b
$$

In type (8), $\mathrm{W}$ is the weighted matrix. And now the question is: how can we get the weighted matrix?

Provided that in the process of electromagnetic wave propagation, the iBeacon signal has received the interference of AWGN, and the AWGN can be represented as:

$N\left(0, \sigma^{2}\right)$

According to type (1), we can get:

$$
N\left(0, \sigma^{2}\right)+p=p_{0}+10 n\left(\lg \left(d \cdot 10^{N\left(0, \frac{1}{100 n^{2}} \sigma^{2}\right)}\right)\right)
$$

It is obvious that, after the iBeacon signal received the interference of AWGN, the distance between the iBeacon and the mobile terminal is:

$$
d=d \cdot 10 \stackrel{N\left(0, \frac{1}{100 n^{2}} \sigma^{2}\right)}{ }
$$




$$
D\left(d^{\prime}\right)=d^{2} \cdot\left(e^{[\sigma \ln (10)]^{2}}-e^{\left[\sigma 0 n^{2}\right.}{ }^{100 n^{2}}\right)
$$

From type (9), we know that, D (d') is only affected by d2. The uncertainty of d' reflect on the variance of D (d'), when the variance is large, the uncertainty of d' is higher. If the uncertainty of d' is higher, we must decrease the weight of d'. So the weighted matrix can be represented as follow:

$$
\left[\begin{array}{cccc}
\frac{1}{d_{1}^{2}} & 0 & 0 & 0 \\
0 & \frac{1}{d_{2}^{2}} & 0 & 0 \\
0 & 0 & \cdots \cdots & 0 \\
0 & 0 & 0 & \frac{1}{d_{n}^{2}}
\end{array}\right]
$$

\section{Introduction of PDR}

Nowadays, the mobile terminal carries the inertial sensors, such as accelerometer, gyroscope and electronic compass. In the ideal state, we can get the speed of the mobile terminal by integrating the acceleration and get the displacement by integrating the speed. This can't work in practice because the noise will accumulate in these two integrations.

PDR (Pedestrian Dead Reckoning) is stated under the above background. Different from traditional inertial positioning method, PDR calculates the step frequency and estimate the step length to get the displacement of pedestrian. Except this, PDR also estimates the direction of the displacement [4].

\section{Step Frequency Algorithm}

When pedestrian walks slowly indoor, the step frequency is about $1.3 \sim 2.5 \mathrm{~Hz}$, while pedestrian walks fast, the step frequency is about $4 \sim 5 \mathrm{~Hz}$. The indoor step frequency of pedestrian seldom exceeds $5 \mathrm{~Hz}$. According to Nyquist sampling theorem, the sampling rate must be two times or higher than signal frequency. In practice, we find that when the sample rate is $20 \mathrm{~Hz}$, the sampling result can perfectly detect step frequency of pedestrian. Higher sampling rate may lead to oversampling, while lower sampling rate may lead to under sampling.

When using inertial sensors to estimate the step frequency of pedestrian, let ax,ay,az represents three axes data of the accelerometer, usually, we use the modulus value of ax,ay,az, to represents acceleration of the pedestrian, so the acceleration of the pedestrian can be represented as follow:

$$
\mathrm{a}=\sqrt{\mathrm{a}_{\mathrm{x}}^{2}+\mathrm{a}_{\mathrm{y}}^{2}+\mathrm{a}_{\mathrm{z}}^{2}}
$$

In the corridor of 4th Teaching Building, Beijing University of Posts and Telecommunications, we use iPad mini3 to collect the acceleration data. The acceleration data can be seen at Figure.3:

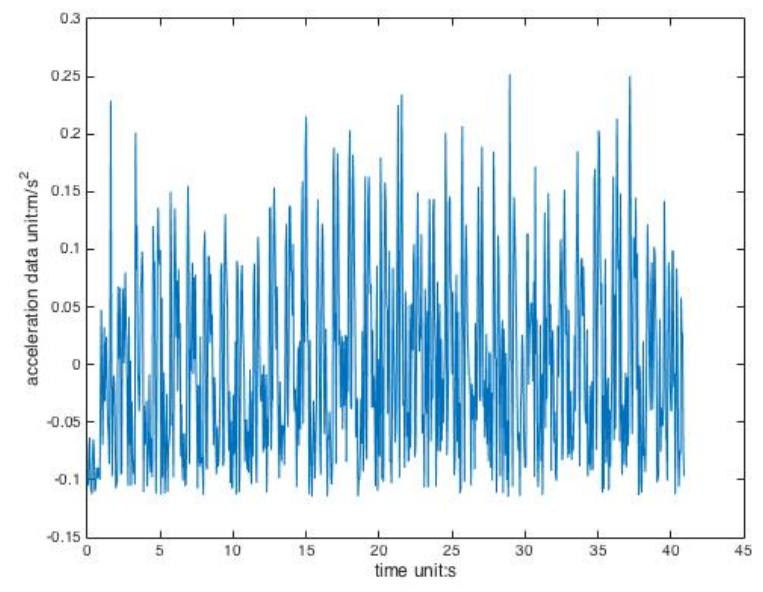

Fig.3. Acceleration data 
It is hard to detect the step frequency because the noise has polluted the useful step frequency. FFT transform of the acceleration data can be seen in Figure.5.

\section{FIR Filter Design}

FIR (finite impulse response filter), is also called non-recursive filter. The output of FIR only depends on current input $x(n)$ and a finite number of past input $x(n-1) \ldots x(n-N+1)$, regardless of past output, thus FIR filter is always stable.

According to the feature of the pedestrian walking indoor, the parameter of FIR filter can be set as follow: pass-band frequency $w p=5 \mathrm{~Hz}$, stop-band frequency ws $=10 \mathrm{~Hz}$, transitional bandwidth tr_width $=5 \mathrm{~Hz}$, we use Kaiser Window as our filter window, the width of Kaiser Window $\mathrm{N}=$ ceil $\left(\left(\right.\right.$ As-7.95)/ $\left(14.36 * t r \_w i d t h /(2 *\right.$ pi $\left.\left.)+1\right)\right)+1=5$. The FIR filter result can be seen in Figure 4

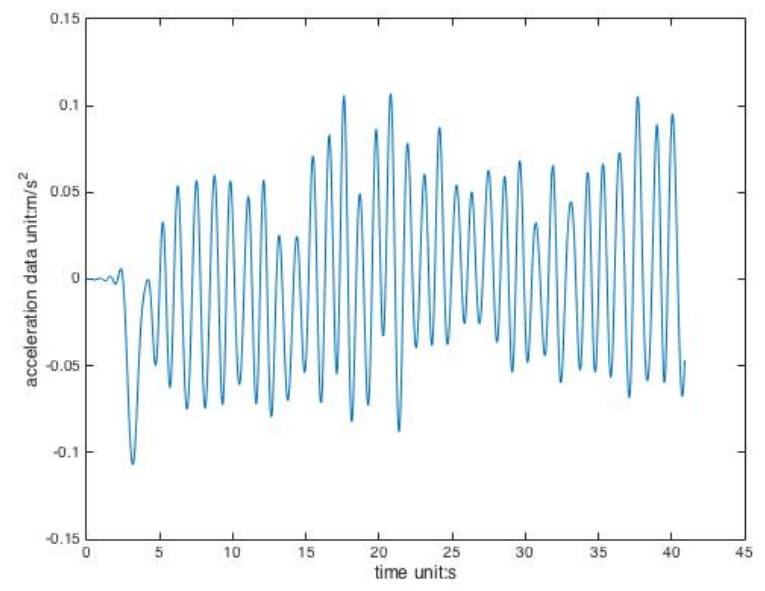

Fig.4. Acceleration data after FIR filter

High-frequency component has been filtered, and "two wave crest" disappear. FFT transform of the acceleration data after FIR filter can be seen in Figure.5:

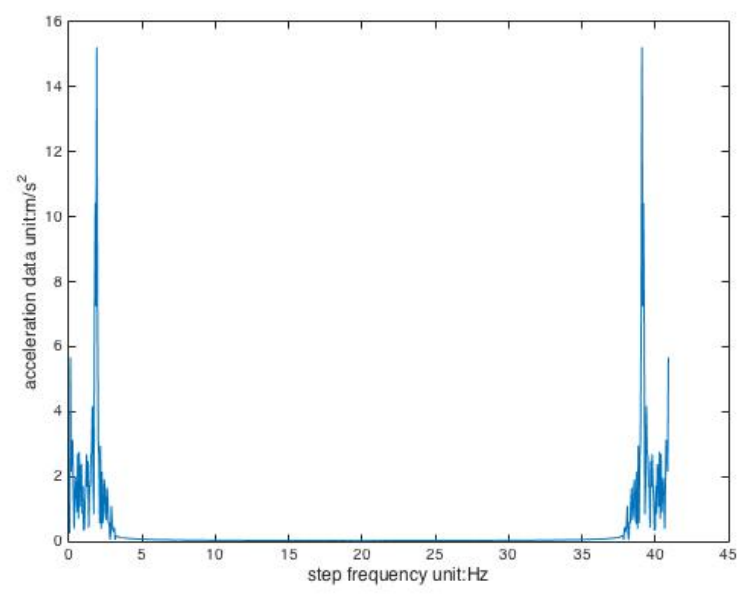

Fig.5. FFT of Acceleration data after FIR filter

High-frequency component has been filtered and the useful step frequency is retained.

\section{Step Frequency Detection Algorithm Based on FIR filter}

As it is stated above, FIR filter can effectively filter the acceleration data. The step frequency detection algorithm based on FIR filter can be represented as Figure.6: 


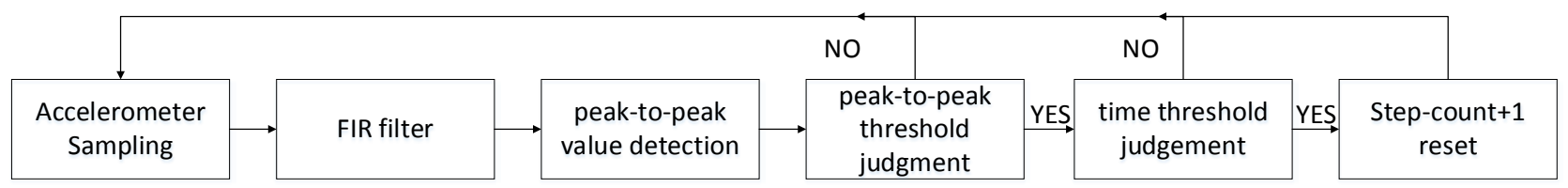

Fig.6. Step frequency Detection Algorithm based on FIR filter

\section{Step Frequency Detection Experiment}

In the corridor of 4th Teaching Building, Beijing University of Posts and Telecommunications, we use iPad mini3 to collect the acceleration data. The experiment can be seen in Table 1:

Table.1

\begin{tabular}{|c|c|c|c|}
\hline Experiment Number & 1 & 2 & 3 \\
\hline Detection number of steps & 87 & 95 & 63 \\
\hline Real number of steps & 86 & 96 & 65 \\
\hline Deviation & $+1.1 \%$ & $-1.04 \%$ & $-3.07 \%$ \\
\hline
\end{tabular}

From Table.1 we know that the step frequency detection algorithm is practicable, the deviation within $3 \%$.

\section{Step frequency Detection Experiment}

Step length detection algorithm is very mature, such as Artificial Intelligence Step Length Estimation Model, Linear Step Estimation Model, and Non-Linear Step Estimation Model. The nature of these algorithms is to find the statistical relationship between step length and step frequency and pedestrian characteristics (such as height and age characteristics) to establish a model, then use this model to estimate the step length. We use Linear Step Estimation Model to detect the step length. The model can be represented as follow [5]:

$$
s=h \cdot\left(a \cdot f_{\text {step }}+b\right)+c
$$

$\mathrm{H}$ represents height of the pedestrian, fstep represents the step frequency, $\mathrm{a}, \mathrm{b}, \mathrm{c}$ represents the correction parameter.

\section{Simulation of PDR technology and RSSI location algorithm}

The deviation of PDR will be accumulated, and saltus step will appear in the RSSI location algorithm because of the noise. The advantages and disadvantages of these two schemes is complementary to each other. Fusion of these two schemes will make better performance of Indoor Positioning.

Assume that we deploy iBeacons in an indoor environment. The area of the indoor environment is $50 \mathrm{M} * 50 \mathrm{M}$. The nodes are uniformly deployed in the area. This can be represented as Figure 7 :

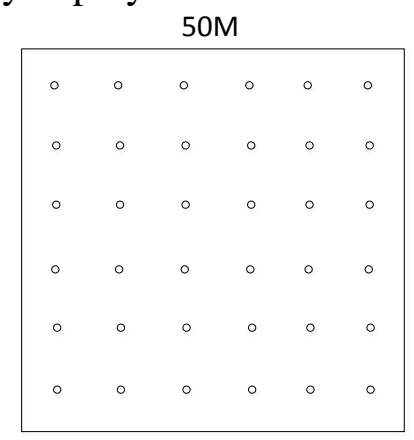

Fig.7.

Provided that iBeacon signal has received interference from AWGN, and the power of AWGN is $-75 \mathrm{~dB}$. The deviation of step length estimation obey the average distribution $[-1,1]$, the deviation of direction estimation obey the average distribution $\left[-10^{\circ}, 10^{\circ}\right]$.

Figure 8 shows the influence of different iBeacon number on the positioning performance of the 
system.

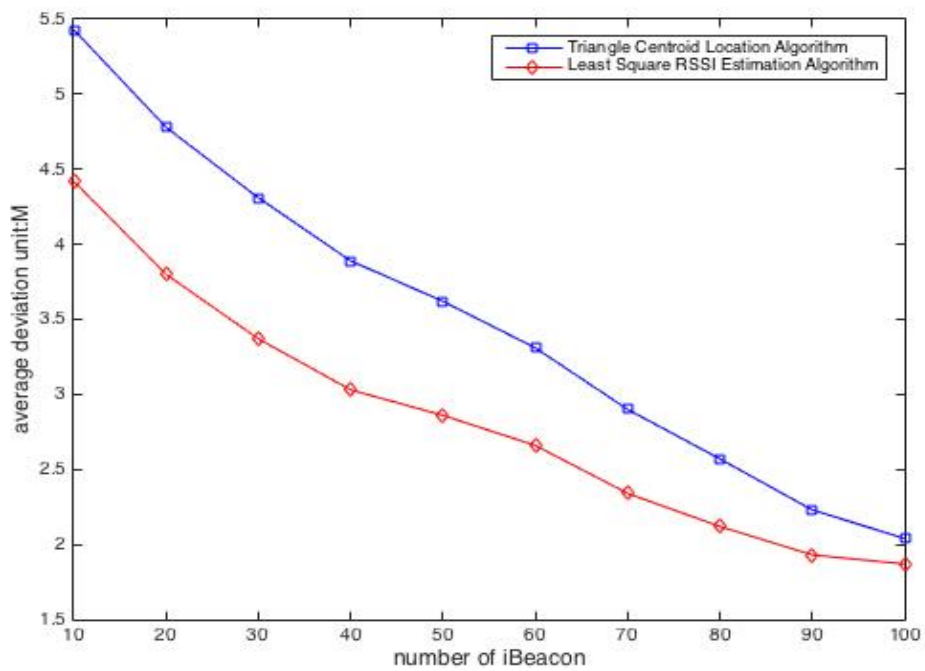

Fig.8.Influence of different iBeacon number on the positioning performance of the system.

\section{Conclusion}

We can find that if the number of iBeacons is 40, the accuracy of Least Squares RSSI Estimation Algorithm is improved by 34\% compared with the accuracy of Triangle Centroid Location Algorithm. When the number of iBeacons is 100, the difference between these two algorithms is tiny. Moreover, in the process of simulation, PDR eliminate the phenomenon of saltus step.

\section{References}

[1] Shi ZhiJing, Xu TieFeng, Liu TaiJun, Liu MingWei: Research on Indoor Positioning Technique Based on iBeacon Base Station. Mobile Terminal, 2011 ,39(7): 55 67.

[2] Lin Wei, Chen ChuanFeng. RSSI-based Triangle and Centroid Location in Wireless Sensor Network. MODERN ELECTRONICS TECHNIQUE,2009， 32(2): 129 130.

[3] Deng ZhongLiang, Yu YanPei, Xu LianMing, Yuan Xie, etc. Indoor and Outdoor Wireless Positioning and Navigation, Beijing University Of Posts And Telecommnnications Press (2013),30 31.

[4] Xu Wei. Research and Implementation of Indoor Positioning Technology based on Android Mobile Phone.2014.

[5] Di WenHua, Liu Yu, Huang ZhenCai. Calculation Scheme and Implementation of Step Length based on iOS platform. ELECTRONIC MEASUREMENT TECHNOLOGY,2012,35(9): 32 35.

[6] Huang Zhen, Li YanWen, Gao Feng. The Expression of the Orientation of a Spatial Moving Unit by Euler Angle. Journal of Yanshan University,2002,26(4): 19 21.

[7] Shi Shuo, Sun Hao, Song Yang. Design of An Experimental Indoor Position System Based on RSSI.Information Science and Engineering (ICISE), 2010 2nd International Conference, 2010: 1989 1992.

[8] Zhao Fang, Luo Haiyong, Geng Hao, Sun Qijin. An RSSI Gradient-based AP Localization Algorithm.Commnications ,China,2014,11(2):100 108. 